\title{
Miescher Granulomatous Macrocheilitis and Management with Triamcinolone Acetonide
}

\author{
Adel Bouguezzi ${ }^{* 1.2}$,Omar Néji ${ }^{1.2}$, Chokri Abdellatif ${ }^{1.2}$, Sameh Sioud ${ }^{1.2}$, Hajer Hentati ${ }^{1.2}$, \\ Jemil Selmi ${ }^{1.2}$ \\ ${ }^{1}$ University of Monastir, Faculty of Dental Medicine, Oral Health and Oro-Facial Rehabilitation \\ Laboratory Research (LR12ES11), Monastir, Tunisia \\ ${ }^{2}$ Dental Clinic of Monastir, Department of Medicine and Oral Surgery, Monastir, Tunisia \\ *Corresponding Author: Adel Bouguezzi, University of Monastir, Faculty of Dental Medicine, Oral \\ Health and Oro-Facial Rehabilitation Laboratory Research (LR12ES11), Monastir, Tunisia
}

\begin{abstract}
Cheilitis granulomatosa $(C G)$ is a chronic swelling of the lip due to granulomatous inflammation. It is a rare inflammatory disorder first described by Miescher in 1945. It is a monosymptomatic form or an incomplete variant of Melkersson-Rosenthal syndrome. As the etiology remains unknown, treatment of CG is challenging. We present a case of CG in a 58-year-old female patient presented to us with diffuse swelling of the lower lip. A histopathological specimen from the lower lip showed non-caseating granulomas. We treated our patient with hydroxychloroquine then intralesional triamcinolone acetonide. The present case highlights the importance of thorough clinical examination, history and investigations in the diagnosis of this lesion as the findings mimic many other granulomatous conditions in dermatology.
\end{abstract}

Keywords: Cheilitis granulomatous, Miescher's cheilitis, Lip.

\section{INTRODUCTION}

Miescher's cheilitis is a chronic disfiguring condition clinically characterized by persistent swelling of the lips, consisting in a granulomatous cheilitis [1]. The etiology of CG is still unknown, but a genetic predisposition may be possible [2]. It presents a series of closely related disease, characterized by lip swelling, such as sarcoidosis, Crohn's disease, foreign body reaction, mycobacterial infections (tubercolosis, leprosy and atypical mycobacteria infection), deep fungal infection, contact allergy and dental infections. Therefore, diagnosis of $\mathrm{CG}$ is a diagnosis of exclusion and all major pathologies that can mimic this condition should be rule out to start the therapy, avoiding the exacerbation of the disease itself [3].

\section{OBSERVATION}

A 58-year-old female patient was referred for a chronic swelling of the lower lip that had appeared several months ago with permanent and radiating pain. The clinical examination revealed a generalized swelling of the lower lip with slight peeling (Figure 1). No other mucosal lesions (Figure 2) or facial paralysis were found. A treatment of prednisolone and metronidazole for 1 month was started without success by his general practitioner. A biopsy was performed (Figure 3) and histological examination showed numerous dilated lymphatic vessels and a mild inflammatory infiltrate, composed of lymphocytes and rare non caseating epithelioid granulomas in the subepithelial connective tissue. Biological, paraclinical examinations and the etiological assessment did not allow to retain enough elements to evoke Crohn's disease, allergic edema or other systemic diseases such as sarcoidosis or lupus. Elements of biological autoimmunity with anti-nuclear antibodies at the threshold of positivity with specificity anti SM and anti RNP were however found.

The provisional diagnosis of Miescher granulomatosis cheilitis was retained. Because of this immunological field, a treatment with hydroxychloroquine (Plaquenil® 200mg) was instituted allowing after 3 months a slight reduction in the lip swelling not satisfactory for the patient. It was then decided to inject triamcinolone acetonide $40 \mathrm{mg}$ retard (Kenacort ${ }^{\circledR}$ ) every 2 weeks in order to obtain a complete remission of the lesion.We noticed a significant improvement in the labial swelling after 1 month of treatment.

In the absence of new signs in favor of SMR or Crohn's disease, the definitive diagnosis of CGM was retained. 


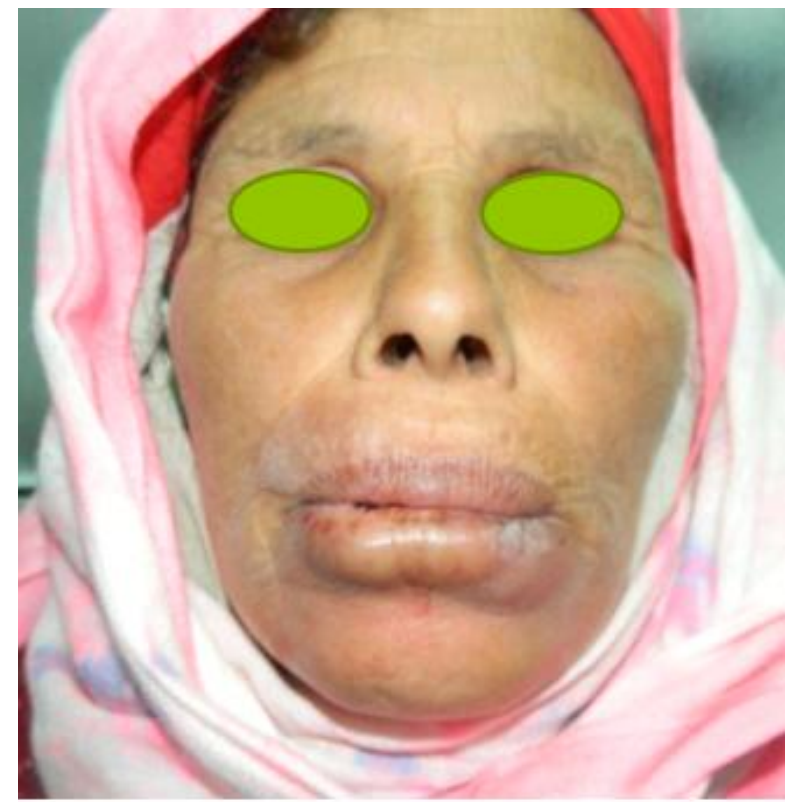

Figure1. Massive enlargement and protuberance of the lower lip

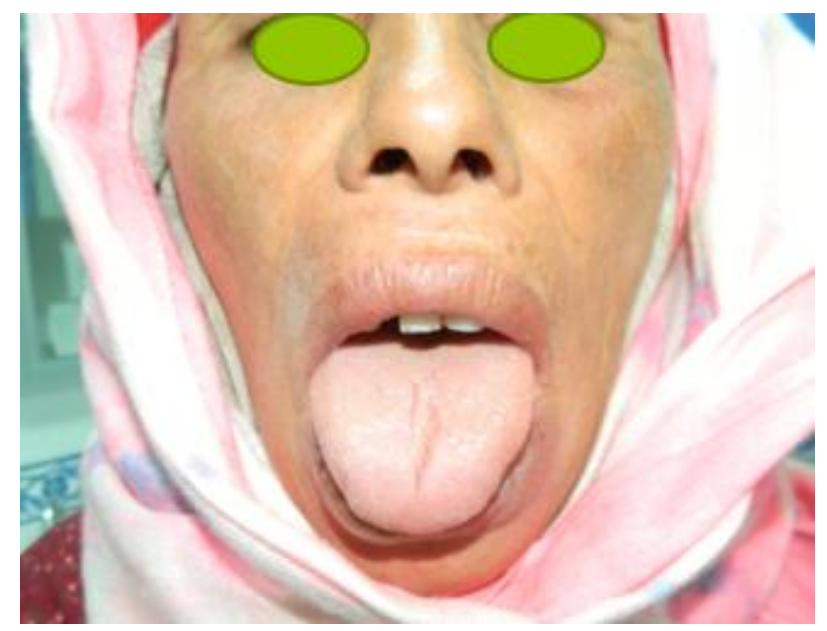

Figure2. Insignificant fissuring in the midline of the tongue

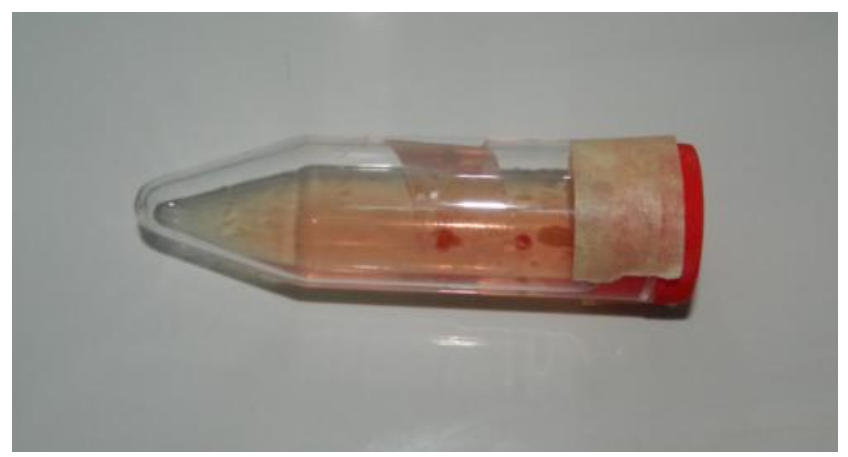

Figure3. Biopsy of labial mucosa

\section{DISCUSSION}

Granulomatous cheilitis is clinically characterized by diffuse and painless swelling of the lips. [4]. When associated with fissured tongue and facial palsy it is known as Melkersson-Rosenthal syndrome More Details $[4,5]$. Isolated granulomatous cheilitis, also known as Miescher granulomatous cheilitis, is sometimes considered a monosymptomatic variant of Melkersson-Rosenthal syndrome [4]. Histopathological features consist of epitheloid cell granulomas with Langhans giant cell in a mononuclear inflammatory background [6].

The diagnostic difficulty lies in the fact that CGM can be an oral manifestation of a systemic disease such as sarcoidosis, Crohn's disease, 
more rarely Wegener's disease or tuberculosis. The histopathological lesion is characterized by the presence of giganto-cellular epithelioid granulomas without caseous necrosis. Due to ignorance of the etiology, many treatments have been proposed such as clofazine, monoclonal antibodies, corticosteroids per os or intralesional injections of corticosteroids which have proved effective in our clinical case. Cosmetic plastic surgery may be offered in the event of medication treatment failures or sequelae. [7].

The differential diagnosis of acquired swelling of the lips are as following Acute causes - trauma, insect bite reaction, angioedema, anaphylaxis, drug reaction, erythema multiforme, urticaria. And chronic causes - Cheilitis granulomatosa, Sarcoidosis, Crohn disease, MelkerssonRosenthal syndrome, Leprosy, Leishmaniasis, Syphilis, Tuberculosis, Rhinoscleroma, Malignancy, Actinic cheilitis, Cheilitis glandularis, Acromegaly, Amyloidosis, $\mathrm{Cl}$ esterase deficiency, foreign body granuloma [8].

Due to its extreme rarity, Miescher's cheilitis receives poor attention and may often remain misdiagnosed. Even if it is very difficult to correlate Miescher's cheilitis to specific etiologic factors, probably the disorder is a polyetiological disease based on genetic or acquired predisposition, associated to functional disturbance of autonomous nervous system resulting in a granulomatous reaction [9]. It may mimics angioedema but it does not respond to antihistamines or steroids. Although it may resolve spontaneously, after several recurrences it could become chronic, resulting in multiple non-infective and non-necrotic granulomas.

The rarity of this disease explains the empiricism of therapeutic proposals and the absence of controlled studies. An effective medical treatment is not available at present. The proposed symptomatic treatments are simply intended to avoid or space recurrences, particularly in the edematous stage. The treatment aims to relieve these patients and to improve their quality of life often very disturbed by the unsightly and distressing nature of macrocheilitis and oro-facial edema [10].The spontaneous disappearance of the disease is rare but has been reported.

Corticosteroid therapy is a classic treatment for CG. It may be administered locally, topically or intralesionally, and at times systemic corticosteroid for short courses (prednisolone: 0.3 to $0.7 \mathrm{mg} / \mathrm{kg} / \mathrm{d} ; 25$ to $50 \mathrm{mg} / \mathrm{d}$ ) maybe tried. Other treatments have also been tried for their anti-inflammatory or immunomodulatory effects such as topical tacrolimus, or oral clofazimine, thalidomide, dapsone, and doxycycline, with inconsistent results [11].

Finally, immunosuppressive treatments such as mycophenolate mofetil, and azathioprine, as well as inhibitors of tumor necrosis factor-alpha (TNF-alpha) have been tried, alone or in combination, in isolated cases and small series with inconsistently positive results. Fumaric Acid esters have given good reesults in $50 \%$ of the cases (who were refractory to other modalities).The cheiloplasty reduction is possible when the lesions are fixed and not evolving [12].

\section{CONCLUSION}

Diagnosis of MRS is difficult as it is a rare disease. Its most frequent finding is the edema in the orofacial area. Dentists should keep the MRS in mind for the patients who have recurrent or permanent swelling in lips, and they should refer them to relevant departments for consultation. We recommend that the intralesional triamcinolone injection seems to be an effective remedy for successful and sustained response in granulomatous cheilitis. Further, randomized case control trials are needed for establishing a universally accepted protocol for management of cheilitis granulomatosa.

\section{REFERENCES}

[1] Nazzaro G., Muratori S. Cheilitis granulomatosa associated with lupus eritematosus discoid and treated with methotrexate: report of a case. An. Bras. Dermatol. 2015;90(3):200-202

[2] Smeets E., Fryns J.P., Van den Berghe H. Melkersson-Rosenthal syndrome and de novo autosomal $\mathrm{t}(9 ; 21)(\mathrm{pl} \mathrm{1;} \mathrm{pl1)} \mathrm{translocation.} \mathrm{Clin.}$ Genet. 1994;45:323

[3] El-Hakim, Chauvin Orofacial granulomatosis presenting as lip swelling. J. Oral Maxillofac. Surg. 2004;62:1114-1117.

[4] Ghorbel IB, SioudDhrif A, Lamloum M, Trabelsi S, Habib Houman M. MelkerssonRosenthal syndrome: Report of 5 cases and review of the literature. Tunis Med. 2006; 84:816-20.

[5] Allen CM, Camisa C, Hamzeh S, Stephens L. Cheilitis granulomatosa: Report of six cases and review of literature. J Am Acad Dermatol. 1990;23:444-50.

[6] Wiesenfield D, Ferguson MM, Mitchell DN, MacDonald DG, Scully C, Cochran K, et al. Oro-facial granulomatosis: A clinical and pathological analysis.Q J Med. 1985;54:101-13. 
[7] Dar N.R, Raza N., Nadeem A, Manzoor A. Granulomatous cheilitis: sustained response to combination of intralesional steroids, metronidazole and minocycline. Journal of the College of Physicians and Surgeons-Pakistan, 2007. vol. 17, pp. 566-567

[8] Park HS, Park ES, Park KC, Cho KH, Youn SW. Chronic idiopathic macrocheilia associated with simple lip enlargement and salivary gland hyperplasia. J Dermatol. 2008;35:234-7.

[9] Ridder G.J., Fradis M., Löhle E. Cheilitis granulomatosa Miescher: treatment with clofazimine and review of the literature. Ann. Otol. Rhinol. Laryngol. 2001;110(10):964-967.
[10] Antoszczyk G, Obtułowicz A, Czarnobilska E, Wojas-Pelc' A. Melkersson-Rosenthal syndrome--diagnostic and therapeutic problems. Prz. Lek. 2008;65(9):390-2

[11] LeBoit PE. From Sweet to Miescher and back again. Am J Dermatopathol. 2006 Aug; 28(4): 381-3

[12] Grinspan D, Borda JM, Casalá A, Díaz J, Abulafia J. [Melkersson-Rosenthal's syndrome. Its relation to Miescher granulomatous macrocheilia and Bernier-Boeck-Schaumann disease]. Arch Argent Dermatol. 1967 Sep; 17(3):237-68

Citation: Adel Bouguezzi et al., "Miescher Granulomatous Macrocheilitis and Management with Triamcinolone Acetonide”, International Journal of Research Studies in Medical and Health Sciences. 2020; 5(6): 12-15.

Copyright: (C) 2020 Adel Bouguezzi et al., This is an open-access article distributed under the terms of the Creative Commons Attribution License, which permits unrestricted use, distribution, and reproduction in any medium, provided the original author and source are credited. 